\title{
Inhibition of acetyl-CoA carboxylase by PP-7a exerts beneficial effects on metabolic dysregulation in a mouse model of diet-induced obesity
}

\author{
TIANYA LIU ${ }^{1,2}$, LINGSHAN GOU $^{3}$, SHIRONG YAN $^{4}$ and TONGHUI HUANG ${ }^{4}$
}

${ }^{1}$ Department of Pharmacy, The Affiliated Hospital of Xuzhou Medical University; ${ }^{2}$ Jiangsu Province Key Laboratory of
Anesthesiology, School of Anesthesiology, Xuzhou Medical University; ${ }^{3}$ Center for Genetic Medicine, Maternity and
Child Health Care Hospital Affiliated to Xuzhou Medical University; ${ }^{4}$ Jiangsu Province Key Laboratory of New Drug
Research and Clinical Pharmacy, School of Pharmacy, Xuzhou Medical University, Xuzhou, Jiangsu 221004, P.R. China

Received July 26, 2019; Accepted March 18, 2020

DOI: $10.3892 /$ etm.2020.8700

\begin{abstract}
Acetyl-coenzyme A carboxylase (ACC) is a critical regulator of fatty acid metabolism and represents a promising therapeutic target for metabolic diseases, including obesity, type 2 diabetes and non-alcoholic fatty liver disease. Recently, a novel ACC inhibitor, PP-7a, was developed by our group by utilizing a structure-based drug design. In the present study, the pharmacological effects of PP-7a on the metabolic dysregulation in mice with high-fat diet (HFD)-induced obesity and the underlying mechanisms were investigated. The inhibitory effect on ACC activities was confirmed by assessing the level of malonyl-CoA, a product synthesized by the catalyzation of ACC. Following 16 weeks of being fed an HFD, the mice were administered PP-7a $(15,45$ or $75 \mathrm{mg} / \mathrm{kg})$ for 4 weeks. The effects of PP-7a on weight gain, glucose intolerance, hepatic lipid accumulation and the increase of serum triglyceride (TG), total cholesterol (TC) and free fatty acids (FFA) in mice were assessed. CP-640186 was used as a positive control drug and administered in the same manner as PP-7a. Chronic administration of PP-7a lowered the malonyl-CoA levels in liver and heart tissues of mice in the HFD group. In addition, HFD-induced weight gain and glucose intolerance were improved by PP-7a treatment in the mice fed the HFD. Furthermore, PP-7a suppressed hepatic lipid accumulation and the increase in TG, TC and FFA levels. Taken together, these results suggest that ACC inhibition by PP-7a may have a beneficial effect on metabolic dysregulation in obese mice.
\end{abstract}

Correspondence to: Dr Tonghui Huang, Jiangsu Province Key Laboratory of New Drug Research and Clinical Pharmacy, School of Pharmacy, Xuzhou Medical University, 209 Tongshan Road, Xuzhou, Jiangsu 221004, P.R. China

E-mail: tonghhuang@xzhmu.edu.cn

Key words: PP-7a, acetyl-coenzyme A carboxylase, high-fat diet-induced obesity, obesity, lipid metabolism

\section{Introduction}

Obesity is associated with multiple comorbidities, including hypertension, sleep apnea, cancers and cardiovascular disease $(1,2)$. In addition, obesity confers an increased risk of developing a number of metabolic diseases, including dyslipidemia, type 2 diabetes and non-alcoholic fatty liver disease (NAFLD) $(3,4)$. Accumulating evidence has indicated that elevated fatty acid synthesis and/or impaired fatty acid oxidation have important roles in the development of obesity and associated metabolic diseases $(5,6)$. Hence, the modulation of fatty acid metabolism may be a potential therapeutic target for obesity-associated metabolic diseases.

Acetyl-CoA carboxylase (ACC) is a biotin-dependent heterodimeric enzyme responsible for the catalyzation of acetyl-CoA to form malonyl-CoA, hence regulating fatty acid metabolism and energy homeostasis (7). Two tissue-specific isozymes of ACC have been identified, including ACC1 and ACC2, which have divergent roles and distinct cellular distributions (4). ACC1, mainly expressed in the liver and adipose tissue, catalyzes the ATP-dependent carboxylation of acetyl-CoA to form malonyl-CoA, which functions as a building block to extend the chain length of fatty acids in liver and adipose $(8,9)$. By contrast, ACC2, abundant in heart and muscle tissues and tethered to the mitochondrial outer membrane, functions as a suppressor of mitochondrial fatty acid oxidation by inhibiting carnitine palmitoyl transferase 1 (CPT-1) $(10,11)$. Located in the mitochondrial outer membrane, CPT-1 assists the transport of long-chain acyl-CoA into the mitochondrial membrane for subsequent $\beta$-oxidation by converting this to acylcarnitines (12). Thus, ACC has an important role in regulating the fatty acid metabolism and energy homeostasis (13). Other studies have demonstrated that the genetic knockout of ACC2 lowers malonyl-CoA levels and enhances fatty acid oxidation in rodent models of dyslipidemia $(14,15)$. In addition, pharmacological inhibition of ACC activity benefits fatty acid metabolism and improves glucose homeostasis in $\mathrm{db} / \mathrm{db}$ mice (16).

Recently, a novel ACC inhibitor targeting ACC carboxyl transfer domain, PP-7a, was developed by our group, which exhibited a potent inhibitory effect on ACC activity in in vitro 
studies $(17,18)$. The present study aimed to evaluate the pharmacological effects of ACC inhibition by PP-7a on metabolic disorders in mice fed a high-fat diet (HFD).

\section{Materials and methods}

Animals and chemical administration. A total of 35 male C57BL/6J mice (age, 6-7 weeks; weight, 18-19g), were obtained from the Branch of National Breeder Center of Rodents (Shanghai, China). They were housed in an environment with controlled temperature $\left(23 \pm 1^{\circ} \mathrm{C}\right)$ and humidity $(55 \pm 5 \%)$, and a 12-h light/dark cycle with free access to water and food. All animal experiments were approved by the Animal Ethics Committee of Xuzhou Medical University (Xuzhou, China) and performed in accordance with the National Institutes of Health Guidelines for the Care and Use of Laboratory Animals. Following one week of acclimation, the mice were randomly divided into a control group and an HFD group. According to the protocol of a previous study (19), the mice in the control group $(\mathrm{n}=6)$ were fed ad libitum a normal chow diet (purchased from the Animal Experimental Center of Xuzhou Medical University) composed with $19 \%$ kcal protein, $68 \%$ kcal carbohydrates and $13 \%$ kcal fat, while the mice of HFD group were fed a HFD consisting of $15 \%$ kcal protein, $43 \%$ kcal carbohydrates and $42 \% \mathrm{kcal}$ fat ad libitum for 16 weeks to develop obesity. The most important parameter of the HFD mouse model is the increased body weight and adipose tissue, as compared with the mice fed a normal chow diet.

Following 16 weeks on the HFD, the mice were randomly divided into five groups, including the HFD ( $\mathrm{n}=6)$, HFD+PP-7a (15 mg/kg, n=5), HFD+PP-7a (45 mg/kg, n=6), HFD+PP-7a ( $75 \mathrm{mg} / \mathrm{kg}, \mathrm{n}=6)$ and HFD+CP-640186 groups $(75 \mathrm{mg} / \mathrm{kg}, \mathrm{n}=6)$. Compound PP-7a was prepared as detailed in a recent study by our group (17). CP-640186, a known potent ACC inhibitor used as the positive control, was supplied by Selleck Chemicals Co. Ltd (20). PP-7a and CP-640186 have similar chemical structures and exhibited a comparable inhibitory effect on $\mathrm{ACC} 1 / 2$ activity and comparable in vitro cytotoxic activities (17). The doses of PP-7a administered to HFD mice were selected based on in vivo studies investigating the pharmacological effects of CP-640186 published previously (20). PP-7a and CP-640186 were dissolved in $0.5 \%$ carboxymethylcellulose. PP-7a was administered at the corresponding doses by gavage once daily for 4 weeks. The mice from the HFD+CP-640186 group received CP-640186 orally each day for 4 weeks. In parallel, the mice in the control group and HFD group were administered $0.5 \%$ carboxymethylcellulose solution. The body weight was measured once a week. After 4 weeks, the mice were subjected to a glucose tolerance test and MRI. Under anesthesia with chloral hydrate $(10 \%, 500 \mathrm{mg} / \mathrm{kg}$, i.p.), the animals were sacrificed by cervical dislocation and none of them exhibited signs of peritonitis following the administration of $10 \%$ chloral hydrate, as in previous studies $(21,22)$. All of the experimental animals were anesthetized with $10 \%$ chloral hydrate prior to blood sampling and were not allowed to survive after blood sampling. Subsequently, the heart, liver and abdominal adipose tissues were harvested for biochemical and histological analysis.

Glucose tolerance test. To evaluate the effects of ACC inhibition on impaired glucose tolerance in obese mice, glucose tolerance tests were performed after 4 weeks of drug administration. In brief, following a $16-\mathrm{h}$ fast, the mice were intragastrically administered glucose $(2 \mathrm{~g} / \mathrm{kg})$. Blood samples were obtained via the tail vein at $0,30,60$ and 120 min after glucose loading and the blood glucose concentrations were measured using the Bayer Contour Glucose Meter (Bayer AG).

Analysis of serum triglyceride (TG), total cholesterol (TC) and free fatty acid (FFA) levels. The concentrations of TG, TC and FFA in serum collected from retro-orbital exsanguination of the mice were measured with respective assay kits (cat. no. SNM227, SNM226 and SK125-1, respectively; Beijing Baiaolaibo Technology Co., Ltd.). Blood samples were collected and centrifuged at $1,200 \mathrm{xg}$ for $10 \mathrm{~min}$ at $4^{\circ} \mathrm{C}$ to collect serum. The serum samples were then added to designated ELISA plates and incubated for $30 \mathrm{~min}$ at $37^{\circ} \mathrm{C}$. After discarding the liquid and drying, each well was washed with wash buffer 5 times. Following the addition of horseradish peroxidase-conjugated reagent, the ELISA plate incubated for $30 \mathrm{~min}$ at $37^{\circ} \mathrm{C}$. After washing with wash buffer, chromogen solution was added to each well for chromogenic reaction for $15 \mathrm{~min}$ at $37^{\circ} \mathrm{C}$ and placed in the dark. Following the addition of stop solution, the absorbance was then measured at $450 \mathrm{~nm}$ using a microplate reader (Thermo Fisher Scientific, Inc.).

Measurement of malonyl-CoA levels. The livers (ACC1-rich tissue) and hearts (ACC2-rich tissue) were harvested and stored at $-80^{\circ} \mathrm{C}$ for the detection of malonyl-CoA levels. The liver and heart tissues were homogenized with PBS buffer and then centrifuged at $12,000 \mathrm{xg}$ at $4^{\circ} \mathrm{C}$ for $15 \mathrm{~min}$. Subsequently, the supernatant was subjected to the detection of malonyl-CoA with a commercial ELISA kit (cat. no. ARB14412; Beijing Baiaolaibo Technology Co., Ltd.) according to the manufacturer's protocol.

Histological analysis. Partial liver and abdominal adipose tissues from individual mice were post-fixed in $4 \%$ paraformaldehyde at $4^{\circ} \mathrm{C}$ for $48 \mathrm{~h}$, followed by dehydration using graded ethanol and embedding in paraffin (23). The tissues were cut to a thickness of $5 \mu \mathrm{m}$. Subsequently, the tissue sections were stained with hematoxylin for $10 \mathrm{~min}$ and eosin for 2 min at room temperature with Hematoxylin-Eosin staining kit (cat. no. D006-1-1; Nanjing Jiancheng Bio-Engineering Institute Co., Ltd), followed by histological analysis under a light microscope (magnification, $\mathrm{x} 400$ ).

Assessment of adipose mass by MRI. To assess the changes in the adipose tissue compartment, mice were subjected to an MRI scan under anesthesia using a Philips Achieva 1.5 Tesla MRI (Philips Medical Systems B.V.). Images were obtained on a ChemiDoc Touch Imaging System and densitometry was performed using Image Lab Software version 5.2.1 (both Bio-Rad Laboratories, Inc.).

Statistical analysis. Values are expressed as the mean \pm standard error of the mean. Statistical significance was assessed using Student's t-test or one-way analysis of variance followed by Tukey's test using GraphPad version 6.0 software (GraphPad Software, Inc.). P $<0.05$ was considered to indicate a statistically significant difference. 

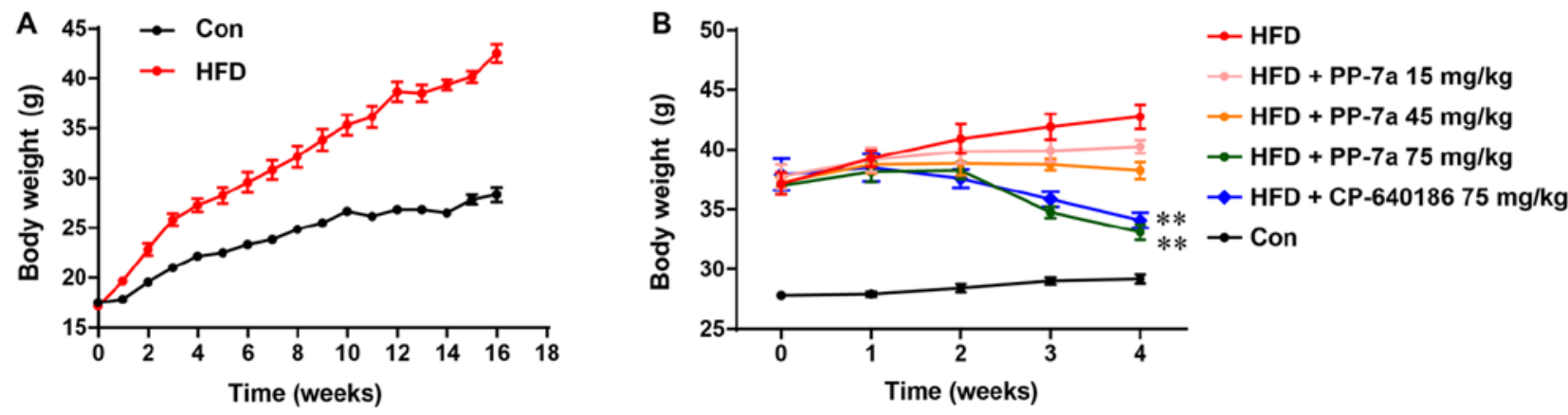

Figure 1. Chronic treatment with PP-7a suppresses weight gain in mice with HFD-induced obesity. (A) Body weight changes in mice fed the chow diet or HFD for 16 weeks. (B) Effect of PP-7a administration on body weight in mice fed the HFD. Mice received PP-7a treatment by gavage at doses of $15,45 \mathrm{or} 75 \mathrm{mg} / \mathrm{kg}$ for 4 weeks. Values are presented as the mean \pm standard error of the mean ( $\mathrm{n}=5-6$ mice per group) ${ }^{* *} \mathrm{P}<0.01 \mathrm{vs.} \mathrm{HFD} \mathrm{group.} \mathrm{HFD,} \mathrm{high-fat} \mathrm{diet;} \mathrm{Con,} \mathrm{control.}$

\section{Results}

PP-7a suppresses the gain of body weight in mice fed an $H F D$. As depicted in Fig. 1A, the body weight of the control mice fed a chow diet or HFD gradually increased during the period of mouse model generation. After 16 weeks on the HFD, the average body weight of mice receiving the HFD increased $>10 \mathrm{~g}$ compared with that of the mice fed the normal chow diet (Fig. 1A) and the changes in body weight were consistent with those reported in a previous study (19). In addition, after 4 weeks of treatment with PP-7a or CP-640186, the weight of the mice in the HFD+PP-7a $(75 \mathrm{mg} / \mathrm{kg})$ group and HFD+CP-640186 $(75 \mathrm{mg} / \mathrm{kg})$ group was significantly decreased, while the weight of the mice in the HFD group without treatment continually increased. Compared with that of the mice in the HFD group, the body weight gain was also suppressed by chronic treatment with PP-7 at doses of 15 and $45 \mathrm{mg} / \mathrm{kg}$ (Fig. 1B). In addition, no toxicity was observed in HFD mice administered PP-7a or CP-640186. In this experiment, visual observations were made for any lethal reactions in HFD mice administered with PP-7a or CP-640186. No such adverse reactions, including a coat with an unhealthy appearance, erythema on the skin surface, appearance of depression or mortality were observed in the HFD mice that had been administered drugs.

Treatment with PP-7a improves glucose tolerance. Glucose tolerance tests were performed to examine the effects of PP-7a on glucose tolerance in mice fed the HFD. Following gavage administration of glucose, all mice in the experimental group displayed elevated blood glucose levels within $120 \mathrm{~min}$. The glucose levels in mice of the HFD group were higher compared with those of the mice in the control group. Chronic treatment with PP-7a exerted a suppressive effect on blood glucose elevation (Fig. 2A and B). PP-7a treatment at $75 \mathrm{mg} / \mathrm{kg}$ produced a glucose-lowering effect comparable to that of the positive control (Fig. 2A and B).

PP-7a suppresses the increase in serum TG, TC and FFA levels in obese mice fed the HFD. Usually, increased serum TG, TC and FFA levels are associated with obesity (24). Thus, to determine the effect of PP-7a on lipid metabolism in the mice fed the HFD, the TG, TC and FFA levels in serum were detected using ELISA kits. As expected, the levels of TG, TC and FFA in the mice fed the HFD were significantly higher than those of the control mice fed a normal chow diet. Chronic administration of PP-7a at the dose of $75 \mathrm{mg} / \mathrm{kg}$ significantly suppressed the increases in the levels of TG and TC (Fig. 3A and B). In addition, PP-7a suppressed the increase in the serum FFA level in a dose-dependent manner (Fig. 3C). There was no significant difference in the levels of serum TG, TC and FFA between HFD+PP-7a $(75 \mathrm{mg} / \mathrm{kg})$ group and the positive control group (Fig. 3A-C).

PP-7a reduces the malonyl-CoA levels in liver and heart tissues of mice fed the HFD. As a key regulator of fatty acid metabolism, malonyl-CoA is synthesized under the catalysis of ACC (25). Hence, the effects of PP-7a on the level of malonyl-CoA in liver and heart tissues were assessed in mice fed the HFD (26). As expected, chronic administration of PP-7a significantly reduced malonyl-CoA levels in liver and heart tissues of mice fed the HFD (Fig. 4A and B). There was no significant difference in the malonyl-CoA levels of liver and heart tissues between HFD+PP-7a $(75 \mathrm{mg} / \mathrm{kg})$ group and the positive control group (Fig. 4A and B).

Hepatic lipogenesis and abdominal adipose accumulation are alleviated by PP-7a. As presented in Fig. 5, the HFD increased liver fat accumulation, which was alleviated by chronic treatment with PP-7a or CP-640186 (Fig. 5A). In addition, histological examination indicated larger adipocytes in the abdominal subcutaneous adipose tissue of the mice fed the HFD compared with the mice fed the chow diet. Compared with the mice fed the HFD, chronic treatment with PP-7a reduced the size of adipocytes (Fig. 5B).

Treatment with PP-7a suppresses fat mass accretion. To determine whether the reduction in body weight was attributed to the decrease of adiposity, quantitative analysis of the abdominal adipose tissue of mice was performed by MRI imaging. As observed from the transverse cross-sections and sagittal sections of MRI images presented in Fig. 6A and B, the abdominal adipose volume in mice from the HFD group was larger compared with that in the control mice. Quantitative evaluation indicated that treatment with PP-7a at the dose of $75 \mathrm{mg} / \mathrm{kg}$ significantly suppressed the increase of abdominal adipose in mice fed the HFD (Fig. 6C and D). No significant 
A

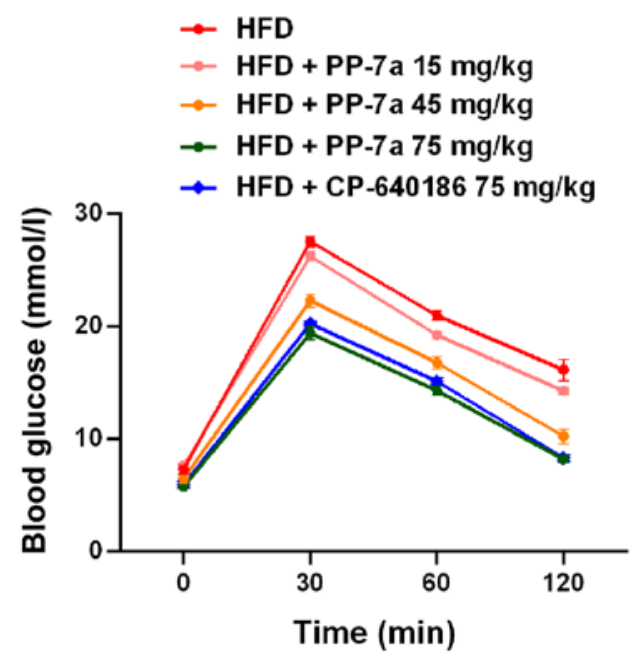

B

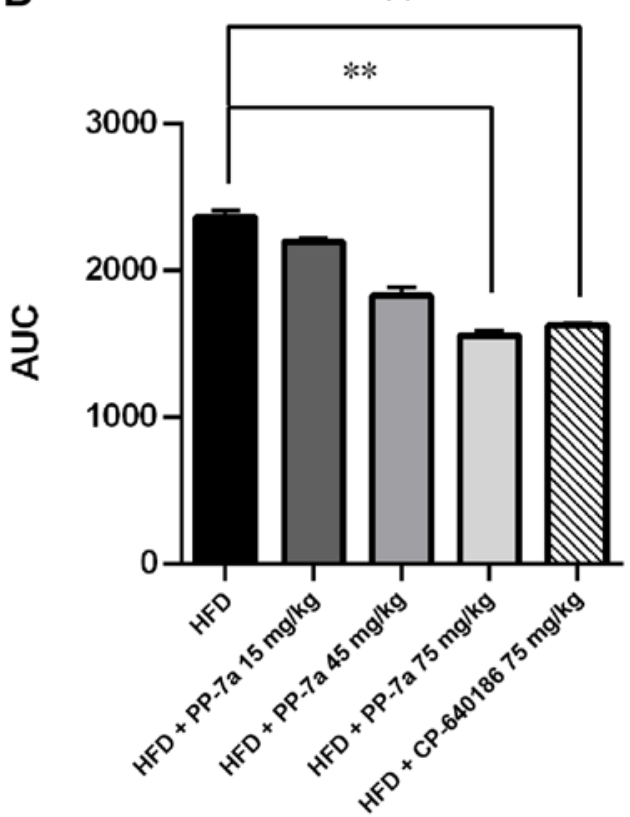

Figure 2. Effects of PP-7a administration on glucose tolerance in mice fed the HFD. (A) Changes in glucose levels in mice following glucose administration within 120 min. (B) AUC values in mice fed the HFD that received PP-7a or CP-640186 treatment in the glucose tolerance test. Values are expressed as the mean \pm standard error of the mean ( $\mathrm{n}=5-6$ mice per group) and analyzed by one-way analysis of variance. ${ }^{* *} \mathrm{P}<0.01$ vs. HFD group. HFD, high-fat diet; AUC, area under the curve.

A

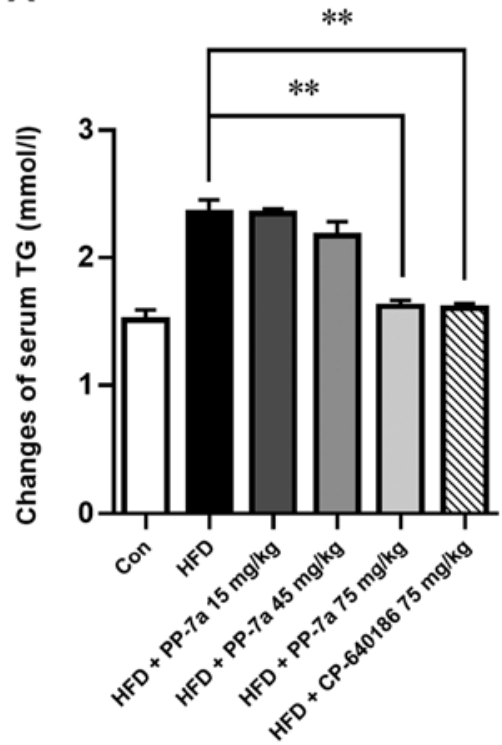

B

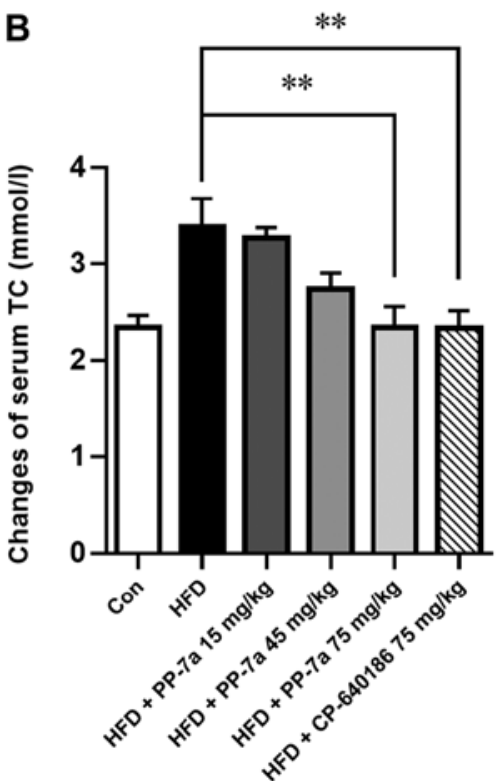

C

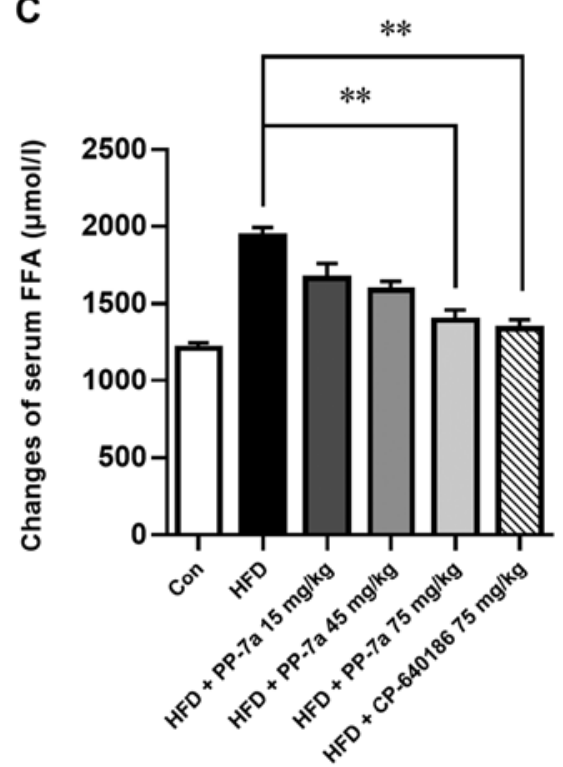

Figure 3. Effect of PP-7a administration on serum TG, TC and FAA levels in mice fed the HFD. Administration of PP-7a (75 mg/kg) for 4 weeks suppressed the increases in serum (A) TG and (B) TC levels in mice fed the HFD. (C) PP-7a suppressed the increases in serum levels of FFA in mice fed the HFD in a dose-dependent manner. Values are expressed as the mean \pm standard error of the mean ( $=5-6$ mice per group) and analyzed by one-way analysis of variance. ${ }^{* *} \mathrm{P}<0.01$ vs. HFD group. TG, triglyceride; TC, total cholesterol; FAA, free fatty acid; HFD, high-fat diet.

difference in the abdominal adipose was observed among HFD+PP-7a (75 mg/kg) group, the positive control group, and the control group (Fig. 6C and D).

\section{Discussion}

ACC functions as a critical regulator of fatty acid biosynthesis and oxidation $(27,28)$. Inhibition of ACC has been reported to exert a suppressive effect on fatty acid biosynthesis, while it promotes fatty acid oxidation $(25,29,30)$. Two isoforms of acetyl-CoA carboxylases, ACC1 and ACC2, exhibit divergent expression profiles and biological functions (31). In a previous study by our group, a novel ACC inhibitor, PP-7a, was developed, which is able to target the carboxyl transfer domain of ACC (17). The present study investigated the pharmacological action of PP-7a to reduce HFD-induced metabolic 
A

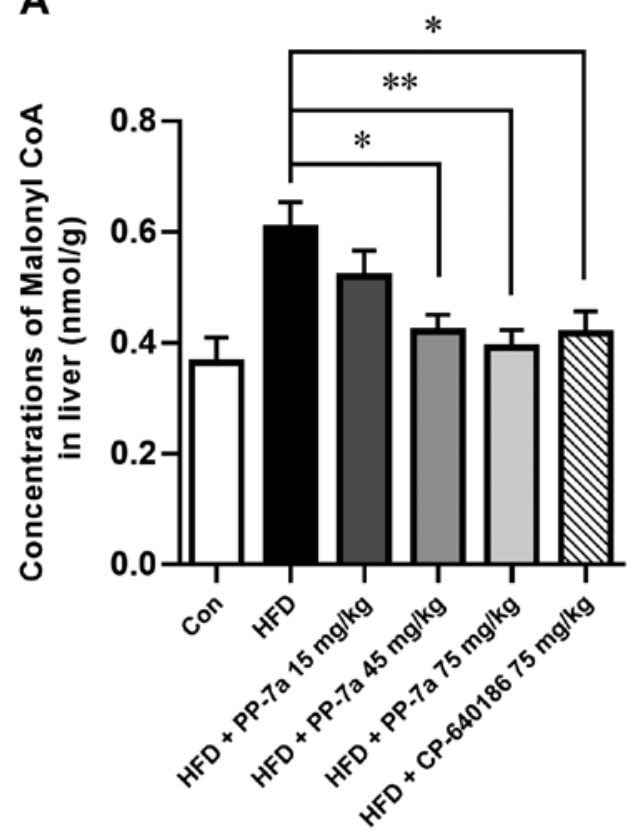

B

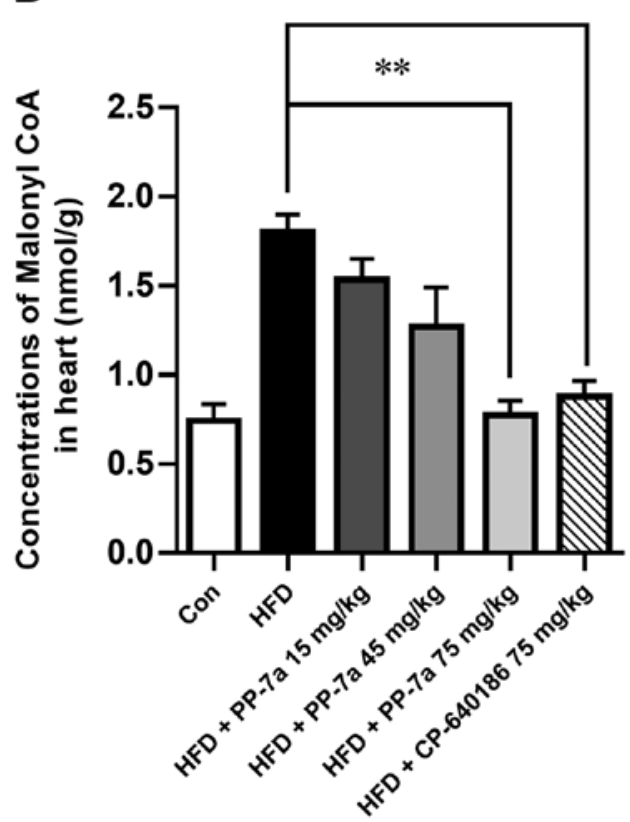

Figure 4. Effect of PP-7a administration on the level of malonyl-CoA in liver and heart tissues of mice fed the HFD. (A) PP-7a treatment decreased the malonyl-CoA content in the livers of HFD mice. (B) PP-7a treatment reduced the malonyl-CoA content in hearts of mice fed the HFD. Values are expressed as the mean \pm standard error of the mean ( $\mathrm{n}=5-6$ mice per group) and analyzed by one-way analysis of variance. ${ }^{*} \mathrm{P}<0.05$ and ${ }^{* *} \mathrm{P}<0.01 \mathrm{vs}$. HFD group. HFD, high-fat diet.

A

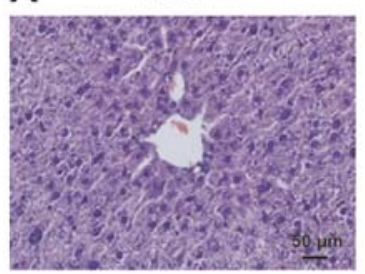

HFD + PP-7a $45 \mathrm{mg} / \mathrm{kg}$

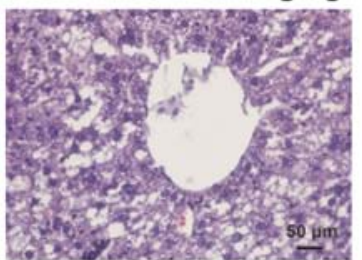

B

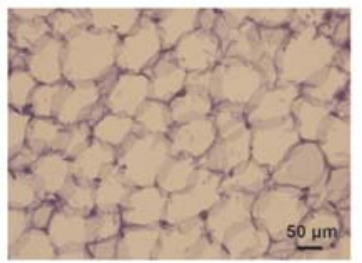

HFD + PP-7a $45 \mathrm{mg} / \mathrm{kg}$

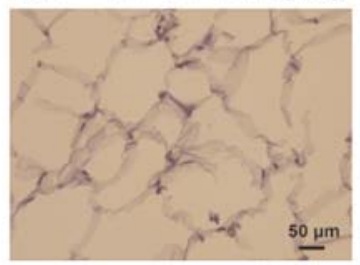

HFD

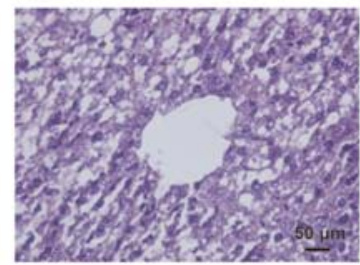

HFD + PP-7a 75 mg/kg HFD + CP-640186 75 mg/kg

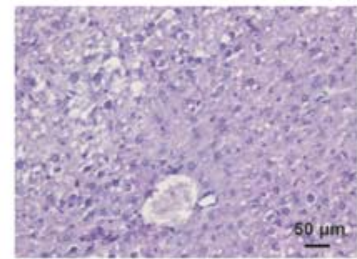

HFD

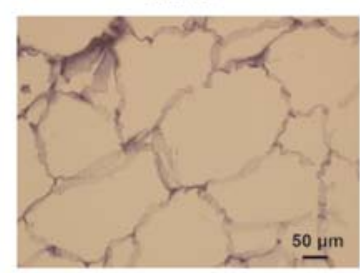

HFD + PP-7a 75 mg/kg HFD + CP-640186 75 mg/kg

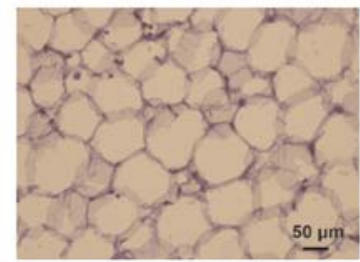

HFD + PP-7a $15 \mathrm{mg} / \mathrm{kg}$
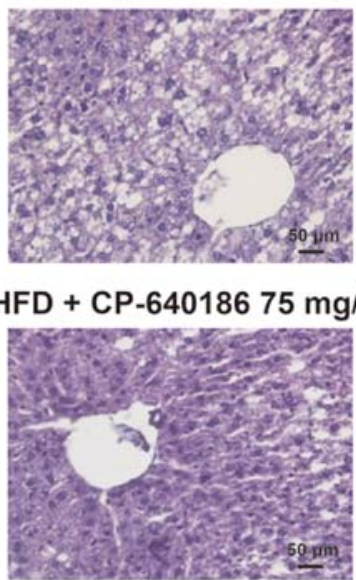

HFD + PP-7a $15 \mathrm{mg} / \mathrm{kg}$
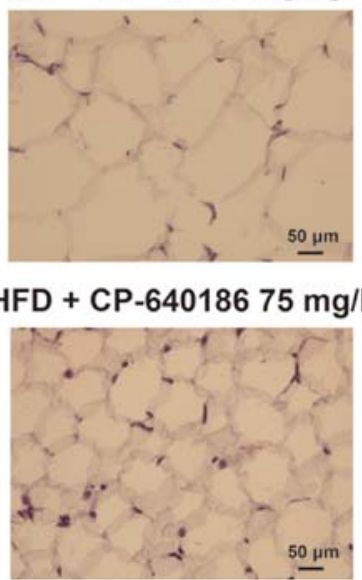

Figure 5. Histological examination of liver and abdominal subcutaneous adipose by H\&E staining. (A) Effect of administration of PP-7a on fat accumulation in the liver of mice fed the HFD. The liver sections were stained with H\&E. (B) Effect of administration of PP-7a on abdominal subcutaneous adipose tissue accumulation in mice fed the HFD. Mice in the HFD group were treated with various doses of PP-7a or the positive control once daily for 4 weeks. The sections of abdominal subcutaneous adipose tissue were stained with $\mathrm{H} \& \mathrm{E}$ (scale bar, $50 \mu \mathrm{m}$ ). $\mathrm{H} \& \mathrm{E}$, hematoxylin and eosin; HFD, high-fat diet. 


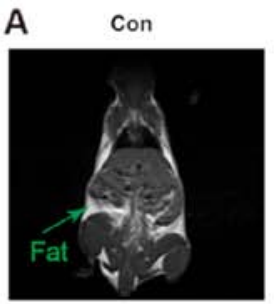

HFD + PP-7a $45 \mathrm{mg} / \mathrm{kg}$

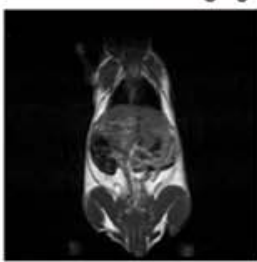

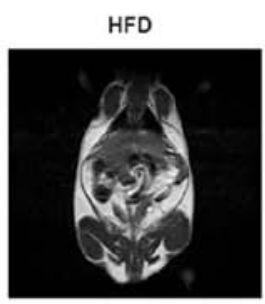

HFD + PP-7a $75 \mathrm{mg} / \mathrm{kg}$

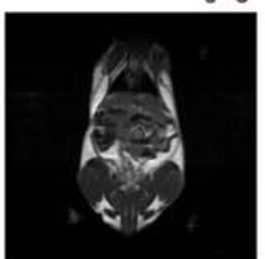

C

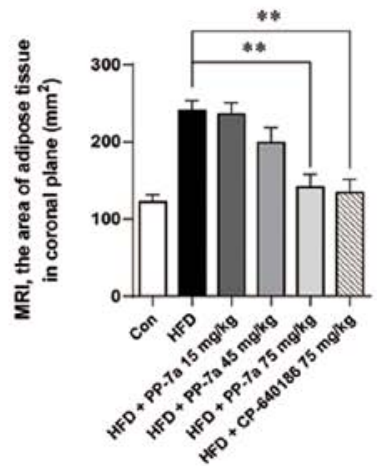

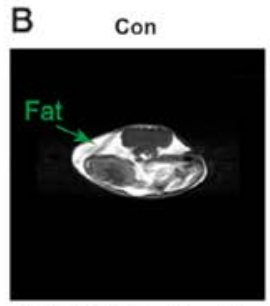
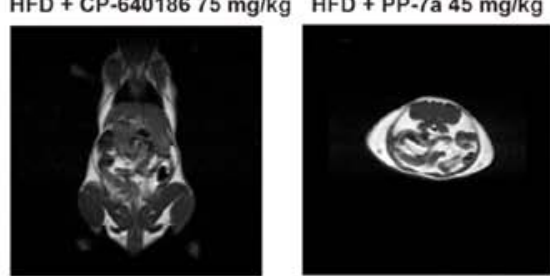

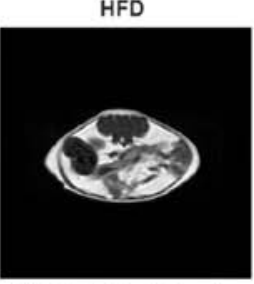

HFD + PP-7a $75 \mathrm{mg} / \mathrm{kg}$

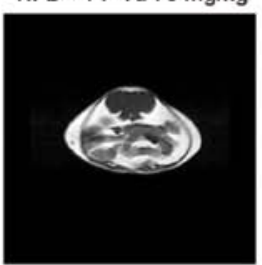

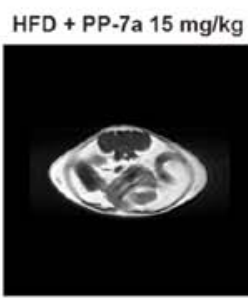

$\mathrm{HFD}+\mathrm{CP}-64018675 \mathrm{mg} / \mathrm{kg}$

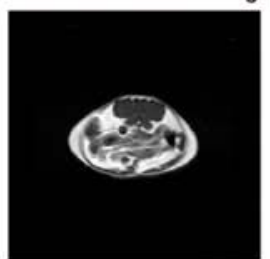

Figure 6. Effect of PP-7a administration on the distribution of adipose tissues in mice fed the HFD. (A) Coronal MRI and (B) transverse MRI of abdominal adipose tissues from each group (fat is indicated by green arrows). (C and D) The area of adipose tissue in each slice from (C) coronal and (D) transverse MRT imaging was determined to calculate the distribution of adipose tissue. Values are expressed as the mean \pm standard error of the mean ( $\mathrm{n}=4-5 \mathrm{mice}$ per group) and analyzed by one-way analysis of variance. ${ }^{*} \mathrm{P}<0.05$ and ${ }^{* *} \mathrm{P}<0.01$ vs. HFD group. HFD, high-fat diet.

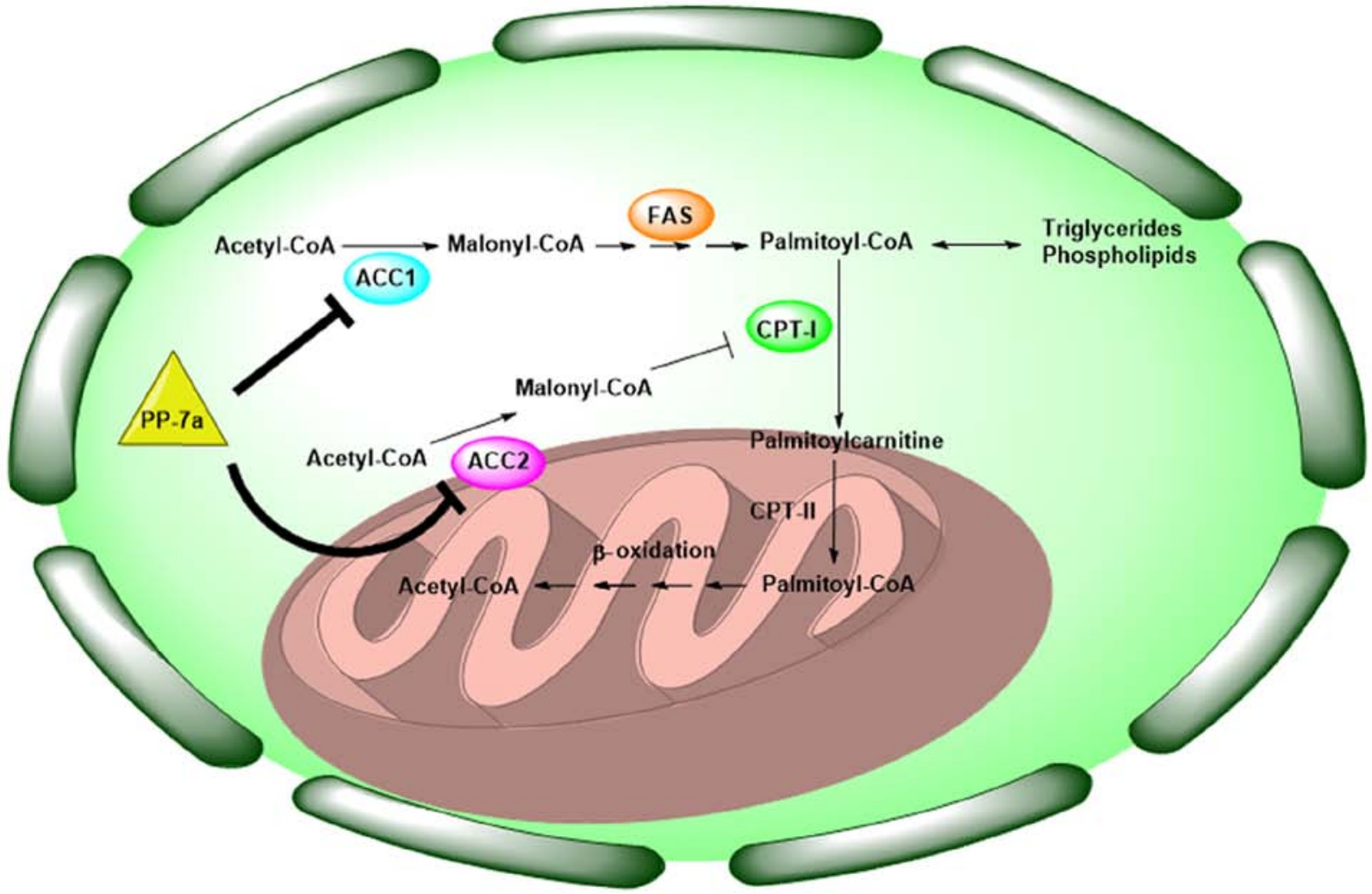

Figure 7. Schematic representing the possible mechanism via which PP-7a, a novel ACC inhibitor, exerts beneficial effects on metabolic dysregulation in mice with HFD-induced obesity. HFD, high-fat diet; ACC, acetyl-coenzyme A carboxylase; CPT-1, carnitine palmitoyl transferase 1. 
dysregulation in mice. There is evidence to suggest that HFD is a major contributor to obesity and associated metabolic syndrome (32). The results of the present study demonstrated that a long-term HFD induced obesity and symptoms of metabolic syndrome, including glucose intolerance, fat accumulation and hyperlipidemia, in mice. These results are consistent with those of previous studies $(33,34)$. However, the weight gain induced by the HFD was suppressed by chronic treatment with PP-7a in a dose-dependent manner at the doses of 15,45 and $75 \mathrm{mg} / \mathrm{kg}$. Furthermore, following treatment with PP-7a at $75 \mathrm{mg} / \mathrm{kg}$ for 4 weeks, the body weight of the HFD-fed mice was significantly decreased. Abdominal MRI further revealed that the mice fed the HFD treated with PP-7a displayed less abdominal fat accumulation compared with the mice treated with the vehicle. These results suggested that decreased fat storage contributed to the weight loss induced by PP-7a.

In addition, the regulation of fatty acid metabolism affects body weight gain and fat accumulation $(7,35)$. The present study indicated that ACC inhibition by PP-7a suppressed HFD-induced fat accumulation. Malonyl-CoA, generating ACC, mediates the regulatory effect of ACC on fatty acid metabolism $(36,37)$. Hence, the malonyl-CoA levels in the liver and heart were detected to confirm the ACC inhibition in the present study. As expected, it was indicated that chronic treatment with PP-7a reduced the malonyl-CoA levels in liver and heart tissues, suggesting a simultaneous inhibitory effect on ACC1 and ACC2 activities. Previous studies have demonstrated that ACC2 deletion enhances ex vivo skeletal muscle fatty acid oxidation and inhibition of ACC2 promotes skeletal muscle fatty acid oxidation in $\mathrm{db} / \mathrm{db}$ mice accompanied by a decrease in the malonyl-CoA level $(16,30)$. However, similar effects were not observed in another study (38). Although the reasons for this discrepancy remains elusive, it may be due to the compensatory increases in fatty acid synthesis mitigated by the ACC2 deletion-mediated efficacy (39). The results of the present study demonstrate that simultaneous inhibition of $\mathrm{ACC} 1$ and $\mathrm{ACC} 2$ by PP-7a resulted in a reduction of malonyl-CoA levels, fat accumulation and weight gain induced by the HFD. In Fig. 7, the hypothesis that PP-7a exerts the beneficial effects on metabolic dysregulation through the inhibition of ACC was rasied, thereby promoting the fatty acid oxidation and inhibiting fatty acid synthesis. The potential mechanisms underlying the pharmacological effects of PP-7a warrant further investigation in the future.

Obesity is associated with hyperlipidemia and glucose intolerance $(40,41)$. In addition, the modulation of fatty acid metabolism affects the plasma lipid levels (42). Hence, in the present study, the effects of chronic treatment with PP-7a on hyperlipidemia in mice with HFD-induced obesity were assessed. As expected, pharmacological inhibition of ACC reduced the levels of TG, TC and FFA in the serum of mice fed the HFD. These results demonstrated that the targeting of ACC by PP-7a has the potential to suppress the increased lipid levels caused by the HFD. Excessive TG levels are the cause of a fatty liver, which contributes to dysregulated glucose metabolism (43). The results of the present study further revealed that PP-7a treatment decreased lipid deposition in the livers of mice fed the HFD. PP-7a administration improved glucose tolerance in HFD-fed mice, suggesting an enhanced effect on glucose homeostasis. Thus, these data support the hypothesis that inhibition of ACC activity suppresses HFD-induced hyperlipidemia and dysregulates glucose homeostasis in mice. However, a limitation of the present study was the lack of ACC expression analysis. The assessment of ACC expression may be helpful to clarify the underlying molecular mechanisms of the pharmacological effect of PP-7a and the effect of PP-7a on the expression of ACC in liver and heart tissues should be examined in a further study.

It should be noted that increased fatty acid oxidation is not always desirable and innocent; hence, the potential adverse effects of ACC inhibition should be considered. Fatty acid synthesis is necessary for T-cell clonal expansion in the immune system and fatty acid oxidation regulates the differentiation of regulatory $\mathrm{T}$ cells, a major regulatory component of the immune system $(44,45)$. Loss of ACC impairs T-cell homeostasis in the periphery and homeostatic proliferation $(46,47)$. In addition, high glucose triggers endothelial injury through increased fatty acid oxidation (48). Other studies have demonstrated that increased free fatty acid levels mediate endothelial dysfunction in subjects with obesity and type 2 diabetes (49-51). Therefore, the present study has a limitation due to the lack of in vivo toxicity tests on PP-7a. More attention should be paid to the side effects of ACC inhibition by ACC inhibitors in future studies. More importantly, the pharmacokinetics of PP-7a should be further explored.

In conclusion, the present study demonstrated that the pharmacological inhibition of ACC by PP-7a, a novel acetyl-CoA carboxylase inhibitor, exerted a beneficial effect on metabolic dysregulation induced by an HFD in mice. In addition, the present study provided further evidence that ACC represents a therapeutic target for the treatment of obesity-associated metabolic diseases.

\section{Acknowledgements}

Not applicable.

\section{Funding}

This study was funded by grants from China Postdoctoral Science Foundation-funded projects (grant nos. 2017M611916 and 2019M661943), the Natural Science Foundation of Jiangsu Province (grant nos. BK20171184 and BK20170258), Jiangsu Planned Projects for Postdoctoral Research Funds (grant no. 1701132C) and National Natural Science Foundation of China (grant no. 81703493).

\section{Availability of data and materials}

The datasets used and/or analyzed during the present study are available from the corresponding author on reasonable request.

\section{Authors' contributions}

THH and TYL designed the current study and wrote the first draft of the manuscript. TYL, LSG and SRY performed the experiments. LSG, SRY and THH collected, analyzed and interpreted the data. TYL, LSG prepared the figures and 
participated in substantive revisions of the important content of the manuscript. All authors read and approved the final manuscript.

\section{Ethics approval and consent to participate}

All animal experiments were performed in compliance with the Chinese legislation on the use and care of laboratory animals and the study was approved by the Ethics Committee on Animal Care and Use of Xuzhou University Medical College (Xuzhou, China).

\section{Patient consent for publication}

Not applicable.

\section{Competing interests}

The authors declare that they have no competing interests.

\section{References}

1. Basen-Engquist $\mathrm{K}$ and Chang M: Obesity and cancer risk: Recent review and evidence. Curr Oncol Rep 13: 71-76, 2011.

2. Gou L, Zhao L, Song W, Wang L, Liu J, Zhang H, Huang Y, Lau CW, Yao X, Tian XY, et al: Inhibition of miR-92a suppresses oxidative stress and improves endothelial function by upregulating heme oxygenase -1 in $\mathrm{db} / \mathrm{db}$ mice. Antioxid Redox Signal 28: 358-370, 2018.

3. Mohammed MS, Sendra S, Lloret J and Bosch I: Systems and WBANs for controlling obesity. J Healthc Eng 2018: 1564748, 2018

4. Tong L and Harwood HJ Jr: Acetyl-coenzyme A carboxylases: Versatile targets for drug discovery. J Cell Biochem 99. 1476-1488, 2006.

5. Harriman G, Greenwood J, Bhat S, Huang X, Wang R, Paul D, Tong L, Saha AK, Westlin WF, Kapeller R and Harwood HJ Jr: Acetyl-CoA carboxylase inhibition by ND-630 reduces hepatic steatosis, improves insulin sensitivity, and modulates dyslipidemia in rats. Proc Natl Acad Sci USA 113: E1796-E1805, 2016.

6. Kim CW, Addy C, Kusunoki J, Anderson NN, Deja S, Fu X, Burgess SC, Li C, Ruddy M, Chakravarthy M, et al: Acetyl CoA carboxylase inhibition reduces Hepatic steatosis but elevates plasma triglycerides in mice and humans: A bedside to bench investigation. Cell Metabol 26: 576, 2017.

7. Wakil SJ and Abu-Elheiga LA: Fatty acid metabolism: Target for metabolic syndrome. J Lipid Res 50 (Suppl): S138-S143, 2009.

8. Harwood HJ Jr: Treating the metabolic syndrome: Acetyl-CoA carboxylase inhibition. Expert Opin Ther Targets 9: 267-281, 2005.

9. Goedeke L, Bates J, Vatner DF, Perry RJ, Wang T, Ramirez R, Li L, Ellis MW, Zhang D, Wong KE, et al: Acetyl-CoA carboxylase inhibition reverses NAFLD and hepatic insulin resistance but promotes hypertriglyceridemia in rodents. Hepatology 68 : 2197-2211, 2018

10. Abu-Elheiga L, Brinkley WR, Zhong L, Chirala SS Woldegiorgis $\mathrm{G}$ and Wakil SJ: The subcellular localization of acetyl-CoA carboxylase 2. Proc Natl Acad Sci USA 97: 1444-1449, 2000.

11. Marin-Garcia $\mathbf{J}$ and Goldenthal MJ: Fatty acid metabolism in cardiac failure: Biochemical, genetic and cellular analysis. Cardiovasc Res 54: 516-527, 2002

12. Qu Q, Zeng F, Liu X, Wang QJ and Deng F: Fatty acid oxidation and carnitine palmitoyltransferase I: Emerging therapeutic targets in cancer. Cell Death Dis 7: e2226, 2016.

13. Ussher JR and Lopaschuk GD: The malonyl CoA axis as a potential target for treating ischaemic heart disease. Cardiovasc Res 79: 259-268, 2008.

14. Baig NA, Herrine SK and Rubin R: Liver disease and diabetes mellitus. Clin Lab Med 21: 193-207, 2001.

15. Abu-Elheiga L, Matzuk MM, Abo-Hashema KA and Wakil SJ: Continuous fatty acid oxidation and reduced fat storage in mice lacking acetyl-CoA carboxylase 2. Science 291: 2613-2616, 2001.
16. Glund S, Schoelch C, Thomas L, Niessen HG, Stiller D, Roth GJ and Neubauer H: Inhibition of acetyl-CoA carboxylase 2 enhances skeletal muscle fatty acid oxidation and improves whole-body glucose homeostasis in $\mathrm{db} / \mathrm{db}$ mice. Diabetologia 55: 2044-2053, 2012.

17. Huang T, Sun J, Wang Q, Gao J and Liu Y: Synthesis, biological evaluation and molecular docking studies of piperidinylpiperidines and spirochromanones Possessing quinoline moieties as Acetyl-CoA carboxylase inhibitors. Molecules 20: 16221-16234, 2015.

18. Huang TH, Sun J, Xu M and Liu Y: Biological evaluation studies of Acetyl-Coa carboxylase inhibitor in high fat diet induced obese mice. Basic Clin Pharmacol 119: 44-45, 2016.

19. Liu Y, Fu X, Lan N, Li S, Zhang J, Wang S, Li C, Shang Y, Huang $T$ and Zhang L: Luteolin protects against high fat diet-induced cognitive deficits in obesity mice. Behav Brain Res 267: 178-188, 2014.

20. Harwood HJ Jr, Petras SF, Shelly LD, Zaccaro LM, Perry DA, Makowski MR, Hargrove DM, Martin KA, Tracey WR, Chapman JG, et al: Isozyme-nonselective N-substituted bipiperidylcarboxamide acetyl-CoA carboxylase inhibitors reduce tissue malonyl-CoA concentrations, inhibit fatty acid synthesis, and increase fatty acid oxidation in cultured cells and in experimental animals. J Biol Chem 278: 37099-37111, 2003.

21. Matsushita H, Johnston MV, Lange MS and Wilson MA: Protective effect of erythropoietin in neonatal hypoxic ischemia in mice. Neuroreport 14: 1757-1761, 2003.

22. Chen B, Ma Y, Xue X, Wei J,Hu G and Lin Y: Tetramethylpyrazine reduces inflammation in the livers of mice fed a high fat diet. Mol Med Rep 19: 2561-2568, 2019.

23. Feng Y, Yu YH, Wang ST, Ren J, Camer D, Hua YZ, Zhang Q, Huang J, Xue DL, Zhang XF, et al: Chlorogenic acid protects $\mathrm{D}$-galactose-induced liver and kidney injury via antioxidation and anti-inflammation effects in mice. Pharm Biol 54: 1027-1034, 2016.

24. Lu JC, Jing J, Yao Q, Fan K, Wang GH, Feng RX, Liang YJ, Chen L, Ge YF and Yao B: Relationship between lipids levels of serum and seminal plasma and semen parameters in 631 Chinese subfertile men. PLoS One 11: e0146304, 2016.

25. Cuthbert KD and Dyck JR: Malonyl-CoA decarboxylase is a major regulator of myocardial fatty acid oxidation. Curr Hypertens Rep 7: 407-411, 2005.

26. Awan MM and Saggerson ED: Malonyl-CoA metabolism in cardiac myocytes and its relevance to the control of fatty acid oxidation. Biochem J 295: 61-66, 1993.

27. Munday MR: Regulation of mammalian acetyl-CoA carboxylase. Biochem Soc Trans 30: 1059-1064, 2002.

28. Saddik M, Gamble J, Witters LA and Lopaschuk GD: Acetyl-CoA carboxylase regulation of fatty acid oxidation in the heart. J Biol Chem 268: 25836-25845, 1993

29. Eleftheriadis T, Pissas G, Sounidaki M, Tsogka K, Antoniadis N, Antoniadi G, Liakopoulos V and Stefanidis I: Indoleamine 2,3-dioxygenase, by degrading L-tryptophan, enhances carnitine palmitoyltransferase I activity and fatty acid oxidation, and exerts fatty acid-dependent effects in human alloreactive CD4+ T-cells. Int J Mol Med 38: 1605-1613, 2016.

30. Choi CS, Savage DB, Abu-Elheiga L, Liu ZX, Kim S, Kulkarni A, Distefano A, Hwang YJ, Reznick RM, Codella R, et al: Continuous fat oxidation in acetyl-CoA carboxylase 2 knockout mice increases total energy expenditure, reduces fat mass, and improves insulin sensitivity. Proc Natl Acad Sci USA 104: 16480-16485, 2007.

31. Strable MS and Ntambi JM: Genetic control of de novo lipogenesis: Role in diet-induced obesity. Crit Rev Biochem Mol Biol 45: 199-214, 2010.

32. Golay A and Bobbioni E: The role of dietary fat in obesity. Int J Obes Relat Metab Disord 21 (Suppl 3): S2-S11, 1997.

33. Heydemann A: An overview of murine high fat diet as a model for type 2 diabetes mellitus. J Diabetes Res 2016: 2902351, 2016.

34. Hariri $\mathrm{N}$ and Thibault L: High-fat diet-induced obesity in animal models. Nutri Res Rev 23: 270-299, 2010.

35. Ronnett GV, Kleman AM, Kim EK, Landree LE and Tu Y: Fatty acid metabolism, the central nervous system, and feeding. Obesity (Silver Spring) 14 (Suppl 5): S201-S207, 2006.

36. Brownsey RW, Boone AN, Elliott JE, Kulpa JE and Lee WM: Regulation of acetyl-CoA carboxylase. Biochem Soc Trans 34: 223-227, 2006.

37. Folmes CD and Lopaschuk GD: Role of malonyl-CoA in heart disease and the hypothalamic control of obesity. Cardiovase Res 73: 278-287, 2007. 
38. Olson DP, Pulinilkunnil T, Cline GW, Shulman GI and Lowell BB: Gene knockout of Acc2 has little effect on body weight, fat mass, or food intake. Proc Natl Acad Sci USA 107: 7598-7603, 2010

39. Hoehn KL, Turner N, Swarbrick MM, Wilks D, Preston E, Phua Y, Joshi H, Furler SM, Larance M, Hegarty BD, et al: Acute or chronic upregulation of mitochondrial fatty acid oxidation has no net effect on whole-body energy expenditure or adiposity. Cell Metab 11: 70-76, 2010.

40. Akiyama T, Tachibana I, Shirohara H, Watanabe $\mathrm{N}$ and Otsuki M High-fat hypercaloric diet induces obesity, glucose intolerance and hyperlipidemia in normal adult male Wistar rat. Diabetes Res Clin Pract 31: 27-35, 1996.

41. Dominiczak MH: Obesity, glucose intolerance and diabetes and their links to cardiovascular disease. Implications for laboratory medicine. Clin Chem Lab Med 41: 1266-1278, 2003.

42. Fernandez ML and West KL: Mechanisms by which dietary fatty acids modulate plasma lipids. J Nutr 135: 2075-2078, 2005.

43. Stefan N, Kantartzis K and Haring HU: Causes and metabolic consequences of Fatty liver. Endocr Rev 29: 939-960, 2008.

44. Eleftheriadis T, Pissas G, Liakopoulos V and Stefanidis I: IDO decreases glycolysis and glutaminolysis by activating GCN2K, while it increases fatty acid oxidation by activating AhR, thus preserving CD4+ T-cell survival and proliferation. Int J Mol Med 42: 557-568, 2018.

45. Eleftheriadis T, Pissas G, Antoniadi G, Liakopoulos V and Stefanidis I: Indoleamine 2,3-dioxygenase depletes tryptophan, activates general control non-derepressible 2 kinase and down-regulates key enzymes involved in fatty acid synthesis in primary human CD4+ T cells. Immunology 146: 292-300, 2015.
46. Byersdorfer CA: The role of Fatty Acid oxidation in the metabolic reprograming of activated t-cells. Front Immunol 5: 641, 2014.

47. Lochner M, Berod L and Sparwasser T: Fatty acid metabolism in the regulation of $\mathrm{T}$ cell function. Trends Immunol 36: 81-91, 2015.

48. Eleftheriadis T, Pissas G, Sounidaki M, Antoniadi G, Rountas C, Liakopoulos V and Stefanidis L: Tryptophan depletion under conditions that imitate insulin resistance enhances fatty acid oxidation and induces endothelial dysfunction through reactive oxygen species-dependent and independent pathways. Mol Cell Biochem 428: 41-56, 2017.

49. Ghosh A, Gao L, Thakur A, Siu PM and Lai CWK: Role of free fatty acids in endothelial dysfunction. J Biomed Sci 24: 50, 2017.

50. de Jongh RT, Serné EH, Ijzerman RG, de Vries G and Stehouwer CD: Free fatty acid levels modulate microvascular function: Relevance for obesity-associated insulin resistance, hypertension, and microangiopathy. Diabetes 53: 2873-2882, 2004.

51. I S Sobczak A, A Blindauer C and J Stewart A: Changes in plasma free fatty acids associated with type-2 diabetes. Nutrients 11: E2022, 2019.

This work is licensed under a Creative Commons Attribution-NonCommercial-NoDerivatives 4.0 International (CC BY-NC-ND 4.0) License. 